\title{
Anabases
}

ANABASES Traditions et réceptions de l'Antiquité

$21 \mid 2015$

Varia

\section{Le monstrum et la gallina : figures féminines dans l' Agamemnon de Stanley}

\section{Marie Saint Martin}

\section{(2) OpenEdition}

1 Journals

Édition électronique

URL : http://journals.openedition.org/anabases/5220

DOI : 10.4000/anabases.5220

ISSN : 2256-9421

Éditeur

E.R.A.S.M.E.

Édition imprimée

Date de publication : 1 avril 2015

Pagination : 45-59

ISSN : 1774-4296

\section{Référence électronique}

Marie Saint Martin, «Le monstrum et la gallina : figures féminines dans l'Agamemnon de Stanley »,

Anabases [En ligne], 21 | 2015, mis en ligne le 01 avril 2018, consulté le 21 octobre 2019. URL : http:// journals.openedition.org/anabases/5220 ; DOI : 10.4000/anabases.5220

(c) Anabases 


\section{Le monstrum et la gallina: figures féminines dans I'Agamemnon de Stanley}

\section{Marie SAINT Martin}

orsque le philologue londonien Thomas Stanley entreprend de rééditer l'œuvre d'Eschyle et d'en donner une traduction latine en ı663, sa démarche s'inscrit dans la continuité du travail de Vettorius, édité chez Estienne en 1557. Les trois éditions précédentes, celle d'Alde Manuce en $5_{51} 8^{2}$, de Turnèbe en $1552^{3}$ et de Robortello ${ }^{4}$ la même année, s'appuyaient sur un manuscrit dans lequel la fin d'Agamemnon était tronquée; la pièce était suivie des Choéphores, dont on ignorait qu'elle en fût indépendante ${ }^{5}$. Vettorius, le premier, rétablit les derniers vers de l'Agamemnon et, surtout, donne aux Choéphores leur indépendance, en

1 Thomas Stanley (I625-1678), issu d'une famille noble et soutien de la cause monarchiste pendant la guerre civile, exerce une activité de poète et de traducteur de textes antiques et modernes (par exemple, de poèmes de Tristan l'Hermite); il est surtout l'auteur d'une History of Philosophy (I655-ı662) qui lui vaut d'être élu charter member de la Royal Society en i66i et fellow en i663. Voir Chiara Tedeschi, Thomas Stanley, editore di Eschilo, Università degli Studi di Trento, 20I0, p. 73-ı08.

2 Alde Manuce, Aischylou tragōdiai hex. Promētheus desmōtēs. Hepta epi Thēbais. Persai. Agamemnōn. Eumenides. Hiketides, Venise, i5ı8.

3 Adrien Turnèbe, Aeschyli tragediae, Paris, 1552.

4 Francesco Robortello, Aeschyli Tragaediae septem, a Francisco Robortello... nunc primum ex manuscriptis libris ab... erratis expurgatae, ac suis metris restitutae, Venise, G. Scottum, 1552 .

5 Le manuscrit sur lequel manque la fin de l'Agamemnon (à partir du vers ir5g) et le début des Choéphores (jusqu'au vers Io) est le Mediceus ou Laurentianus XXXII 9. La fin de l'Agamemnon est transcrite sur le Florentinus XXXI 8, le Venetus 663 et le 
s'appuyant sur un manuscrit plus complet. Une dernière édition, de Gulielmus Canterus, en I58o, apporte peu d'améliorations au texte ${ }^{6}$. Stanley, en s'appuyant sur le travail de Vettorius, donne ce qui va devenir une édition de référence, qui opère la synthèse encyclopédique du travail de ses prédécesseurs: on y trouve les préfaces et commentaires de Turnèbe, Robortello, Vettorius et Gulielmus Canterus. Surtout, la traduction de Stanley fait date, car elle est d'une fidélité rare à l'époque à l'égard du texte d'Eschyle - et sa valeur se mesure à l'aune des traductions du xvI $^{\mathrm{e}}$ siècle, celle de Jean de Saint Ravy ${ }^{7}$ de Montpellier (I556) en particulier, puisque les autres traducteurs d'Eschyle, Coriolano Martirano (Prométhée, i556), Mathias Garbitius (Prométhée, r559) et Florent Chrestien (Les Sept contre Thèbes, ı585), semblent s'être peu intéressés à l'Orestie ${ }^{8}$.

Je me propose ici de mesurer l'originalité de Stanley et de sa version latine à l'aune d'autres traductions d'Eschyle, postérieures, et en français, celles de Lefranc de Pompignan et de Du Theil ${ }^{9}$, afin de montrer combien l'auteur anglais, peut-être parce qu'il se réfère à un modèle britannique et non français, ou parce qu'il traduit en latin, respecte le texte original, qu'il s'agisse de l'ordre des mots, du choix du vocabulaire ou du nombre très restreint d'ajouts - ou, parfois, l'infléchit dans un sens particulièrement original.

Dans un premier temps, j'essaierai d'évaluer l'importance de la source sénéquienne pour Stanley, afin de montrer comment le modèle latin de Sénèque peut l'aider à lire Eschyle. Puis je soulignerai l'attitude ambiguë qui affleure parfois,

Farnesianus I E 5; les premiers vers des Choéphores ont été conservés par Aristophane et les grammairiens.

6 Guillaume Canter, Aeschyli Tragoediae VII, in quibus, praeter infinita menda sublata, carminum omnium ratio, hactenus ignorata, nunc primum proditur, opera Gulielmi Canteri, Anvers, Christophe Plantin, I58o.

7 Jean de Saint Ravy de Montpellier, Aeschyli... Tragoediae sex... e greco in latinum sermonem, pro utriusque linguae tyronibus, ad verbum conversae, per Johannem Sanravium, Bâle, Jean Oporin, I555.

8 Coriolano Martirano, Coriolani Martirani cosentini episcopi Santi Marei Tragoediae VIII: Medea, Electra, Hippolytus, Bacchae, Phoenissae, Cyclops, Prometheus, Christius - Comoediae II: Plutus, Nubes-Odysseae lib. XII-Batracho-myomachia-Argonautica, Naples, J. M. Simonetta de Crémone, I556; Mathias Garbitius, Aeschyli Prometheus, cum interpretatione Mathiae Garbitii, Bâle, Jean Oporin, I559; Florent Chrestien, Aeschyli tragoedia septem Thebana. Septem thebana tragoedia Aeschulea, stylo ad veteres tragicos latinos acredente quam proxime fieripotuit a G. Septimio Florente Christiano, 1585, Paris, Frédéric Morel. Voir A. Wartelle, Histoire du texte d'Eschyle, Paris, Les Belles Lettres, I97I, et M. Mund-Dopchie, La Survie d'Eschyle à la Renaissance, Editions, traductions, commentaires et imitations, Louvain, Peeters, I984.

9 C. Lechevalier, L'Invention d'une origine: Traduire Eschyle en France de Lefranc de Pompignan à Mazon: le Prométhée enchaîné, Paris, Honoré Champion, 2007. 
malgré tout, à l'égard des horreurs montrées par Eschyle ; enfin, je m’interrogerai sur différents choix de lexique suffisamment révélateurs pour être porteurs d'une vision de la famille un peu différente de celle que développe le texte grec.

Dans un premier temps, je voudrais essayer de montrer ce que la traduction de Stanley contient de présupposés poétiques. Le modèle latin auquel le traducteur pouvait le mieux s’identifier est sans aucun doute Sénèque, puisqu'il s'agit du seul tragique qui soit parvenu dans cette langue, et que Sénèque avait écrit lui aussi un Agamemnon; il utilise cependant également des références transposées d'Aristote, et réalise une synthèse originale sur le plan poétique.

La traduction produite par Stanley est d'une fiabilité impressionnante: il n'est que de prendre, par exemple et presque au hasard, les vers prononcés par Cassandre pour prédire sa propre mort. La traduction latine suit mot à mot le texte grec, respectant jusqu'à l'ordre précis des termes dans la phrase:

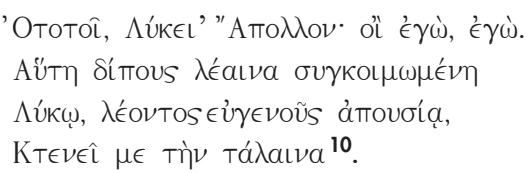

Apollon Lykéios, pitié, pitié pour moi! - C'est elle, la lionne à deux pieds qui dormait avec le loup en l'absence du noble lion, c'est elle qui va me tuer, malheureuse ${ }^{11}$ !

Ototoi, Lycie Apollo! heu me, me!

Haec bipes Leaena commista

Lupo, Leonis generosi absentia,

Me miseram interimet.

Ototoi, Apollon Lycien! malheur à moi, à moi! Cette femme, la lionne à deux pieds, alliée au loup, en l'absence du noble lion, malheureuse que je suis, elle me tuera ${ }^{12}$.

La seule liberté que se permet Stanley, dans ces quatre vers où le rythme même est respecté, est l'inversion du complément me miseram, rejeté devant un verbe

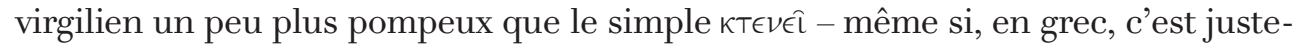
ment l'absence de préfixe qui signale le ton poétique du mot. Notons cependant que cette démonstration de fidélité suit immédiatement un vers où Stanley s'éloigne

10 Thomas Stanley, Agamemnon, dans Aeschyli tragoediae septem cum scholiis graecis omnibus, deperditorum dramatum fragmentis, versione et commentario, Londres, Jacobi Flesher, ı664, v. I266-ı269, p. 38o-38ı.

11 Traduction Paul Mazon, Paris, Les Belles Lettres, I925, v. I257-I26o, p. 55.

12 Je traduis le latin de Stanley. 


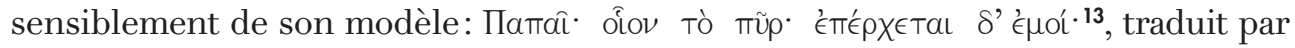
Proh dï! qualis calor corpus meum invadit! Dans ce vers, Stanley fait intervenir une adresse aux dieux pour remplacer l'interjection Патаі̃ (qu'il sait pourtant traduire par papae ailleurs ${ }^{14}$ ), et surtout interprète le feu qui «marche» (pour reprendre la traduction de Paul Mazon) sur Cassandre comme une chaleur qui entre dans son corps. Ces modifications vont dans le sens d'une amplification de la résonance religieuse dans le texte eschyléen, l'image de la chaleur qui envahit le corps pouvant rappeler les images chrétiennes de la foi ${ }^{15}$; par ailleurs, l'appel aux dieux, qui ne se trouve pas dans le grec, et la glose transformant le feu en chaleur intérieure, rendent le vers plus lisible pour un public moderne - plus attendu, et plus faible de ce fait. La dimension pathétique est également amplifiée, notamment parce que l'hallucination de Cassandre est vécue comme une émotion intérieure et non comme un feu venant de l'extérieur. Lorsque Stanley prend quelques libertés avec son modèle, c'est d'ailleurs fréquemment dans le sens d'un pathos accru, au moment de traduire des interjections de ce type: ainsi, au vers 220, Stanley préfère-t-il suivre la leçon de Triclinius, ajoutant un deuxième tí qu'il traduit, d'une manière un peu lourde mais efficace sur le plan dramatique, par la répétition de quodnam ${ }^{16}$. Dans le même passage, $\lambda \iota$ тróvaus (celui qui abandonne son vaisseau) est traduit, comme très souvent pour ces mots composés dont Eschyle raffole, par une glose, ici plus nettement péjorative et morale, proditor classis ${ }^{17}$. Ces redoublements ou amplifications, suffisamment rares pour être notés - la traduction en latin comporte exactement le même nombre de vers que le texte grec édité par Stanley - vont tous dans le sens d'une amplification pathétique.

Or ces aménagements conviennent à une définition de la tragédie bien plus propre à Sénèque qu'à Aristote. La traduction de l'argument rend compte de ces oscillations, résolues par un positionnement du côté du spectaculaire: le passage jugé le plus étonnant par l'argument est celui dans lequel Cassandre vaticine passage qui avait justement disparu des premières éditions: Haec fabulae pars mirabilis est, utpote et ad stuporem et commiserationem permovendam apta. Le verbe $\theta a v \mu a ́ \zeta \epsilon$ бa , utilisé au sujet de ce passage dans le texte grec, est traduit par l'adjectif mirabilis, qui rend bien compte de la portée à la fois étonnante, admirable et peut-être même monstrueuse de la scène dédiée à Cassandre. Il est dit de cette partie étonnante, qu'elle est ad stuporem et commiserationem permovendam apta ${ }^{\mathbf{1 8}}$,

13 Stanley, Agamemnon, v. I265; v. 1256 de l'édition P. Mazon, ibid., qui traduit «Ah! ah! quel est ce feu? Et il marche sur moi...»

14 Stanley, Agamemnon, v. Iเ23, p.37I.

15 Voir Psaumes, XVIII, 7, et saint Augustin, Confessions, V, I.

16 Stanley, Agamemnon, p.3ı8-3ıg.

17 Ibid., v. 22I.

18 Ibid., p.302-3o3. 
“apte à susciter la stupeur et la commisération ». Le balancement peut sembler

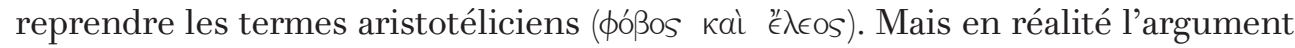

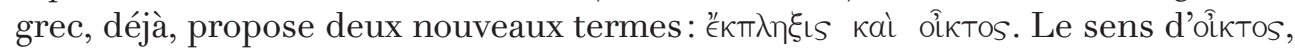
traduit par commiseratio, convient plutôt bien à une doctrine aristotélicienne de

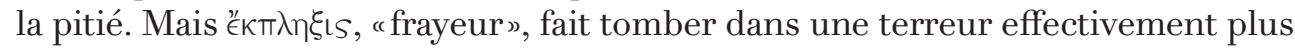
représentative des tragédies de Sénèque ou d'Eschyle, tel qu'il est compris par le XVII ${ }^{\mathrm{e}}$ siècle; surtout, sa traduction par stupor, "saisissement », qui induit davantage

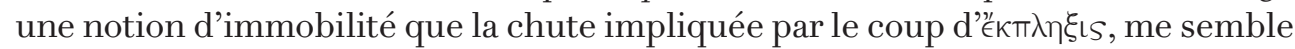
plus proche d'une dramaturgie sénéquienne statique, dans laquelle le monstrum fait naître le stupor, que d'une poétique aristotélicienne des passions tragiques. La suite de l'argument met d'ailleurs l'accent sur la spécificité d'Eschyle, qui, seul (unus pour traduire íścws), fait tuer Agamemnon sur la scène. Cette originalité du dramaturge grec est ce qui le rend incompatible avec la doctrine aristotélicienne du théâtre, mais qui le rapproche de Sénèque: il s'agit du spectacle horrible de la mort sur scène, ce qui explique, peut-être, qu'un anglais comme Stanley soit bien plus prompt à l'accepter que des français réfractaires au modèle shakespearien.

Dans le cours de la pièce, Stanley se laisse très peu aller à des interprétations trop libres du texte d'Eschyle ; il arrive cependant qu'il opère des choix de traduction montrant plus clairement l'influence de Sénèque sur son travail. Le vocabulaire du monstrum et du nefas, identifié par Florence Dupont comme propre à la poétique de Sénèque, affleure de manière parfois surprenante dans la traduction de Stanley. Les vers du premier chœur, consacrés à Iphigénie, soulignent ainsi la naissance du nefas, le crime en horreur aux dieux, qui appelle irrémédiablement le châtiment sur la tête d'Agamemnon:

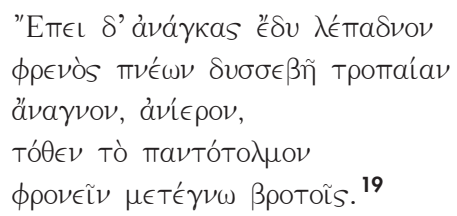

Et, sous son front une fois ployé au joug du destin, un revirement se fait, impur, impie, sacrilège : il est prêt à tout oser, sa résolution désormais est prise. Car, à la source de tous les maux, la funeste démence aux desseins honteux est là pour souffler l'audace aux mortels ${ }^{20}$.

Exinde necessitatis subiit capistrum

Mentis spirans impium ventiversivum, Nefandum, profanum,

19 Ibid., v. 226-23o, p.3ı8-3ıg.

20 Traduction Mazon (v. 2I8-22I dans cette édition), p. I7. 
Unde supra modum

Sapere poenituit mortales.

À partir de là, il se prête à la muselière de la nécessité, respirant un revirement impie, sacrilège, criminel, dont souvent, au-delà de la mesure, les mortels se sont repentis.

La structure du texte fait l'objet d'un calque mot à mot. Mais les adjectifs

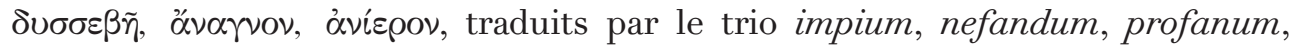
prennent une tournure très proche de certains textes de Sénèque - impius était, dans l'Agamemnon de ce dernier, réservé par Clytemnestre à la flotte libérée par le sacrifice de sa fille ${ }^{21}$, ce dernier événement constituant le premier nefas, destiné à être suivi d'un second, le meurtre d'Agamemnon ${ }^{22}$.

Plus loin, Clytemnestre est qualifiée de monstrum:

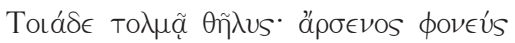

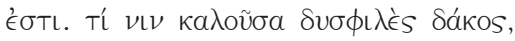

Túxol $\mu^{\prime}$ ă $v^{23}$

Telle est l'effronterie [de cette femelle]; tueuse du mal, je vois en elle... de [quelle odieuse bête sauvage] [...] devrais-je emprunter le nom [...]? ${ }^{24}$

Talia audet foemina; interfectrix est

Viri: quod illam monstrum invisum appellans

Vere appellaverim?

Voici les audaces de cette femme; elle est tueuse d'homme: du nom de quel monstre odieux pourrais-je l'appeler pour l'appeler correctement?

Le terme sákos fait bien référence à une bête qui pourrait mordre, ou à une morsure. Mais le monstrum, lui, plonge le spectateur dans l'univers de l'anormalité et de la perversion, du prodige - et, en l'occurrence, du prodige maléfique d'une femelle tueuse de mâle. Le monstrum vient directement de l'intertexte sénéquien: prodige en horreur aux dieux, Clytemnestre est comparable à Atrée lors du festin qu'il réserve à son frère ${ }^{25}$.

21 Sénèque, Agamemnon, édité par Fr.-R. Chaumartin, Paris, Les Belles Lettres, I999, v. I73. Également v. 2I9.

22 Voir F. Dupont, Les monstres de Sénèque, Paris, Belin, 1995, coll. “L'Antiquité au présent », p. 175-178.

23 Stanley, Agamemnon., v. 1240-1242, p.378-379.

24 Traduction Mazon, v. I23I-I232, p.54, adaptée par moi (la ponctuation retenue est différente).

25 Sénèque, Thyeste, v. 7o3; voir Fl. Dupont, Les monstres de Sénèque, p. 55-go. 
Le dernier indice qui prouve combien Sénèque est présent à l'esprit de Stanley lorsqu'il traduit Eschyle, c'est l'apparition du sentiment non pas de terreur ou de pitié, mais bien d'horreur, suscité par le spectacle du monstrum:

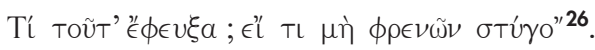

Pourquoi [as-tu pris la fuite]? quel monstre [objet d'horreur] se forge donc ton âme ${ }^{27}$ ?

Quid adeo refugis? nisi aliquis sit mentis horror.

Pourquoi fuis-tu donc? y aurait-il quelque objet d'horreur dans ton âme?

Le terme horror, choisi pour traduire oTúyos, est tout à fait bienvenu; et pris dans l'enchaînement qui mène du nefas au monstrum, puis à l'horror, il confirme les résonances sénéquiennes des autres mots. Notons que le mot est entouré par le фóßos, évoqué dans le vers précédent et dans celui qui suit - mot qui ne peut manquer, pour Stanley comme pour nous, d'évoquer la terreur aristotélicienne. Or, très étonnamment, le choix de maintenir фóßov au vers suivant, selon la leçon de Triclinius, n'est pas suivi en traduction, puisque ce mot est traduit par caedem, comme s'il traduisait le фóvov proposé par les autres manuscrits. Au vers i3ı5, il est cette fois traduit par metus, là où l'on aurait plutôt attendu timor - or metus est le mot utilisé par Sénèque pour traduire les angoisses de Clytemnestre ${ }^{28}$.

Le texte produit par Stanley, tout en demeurant très proche de celui d'Eschyle, transforme le modèle en une œuvre que l'on pourrait qualifier de sénéquienne. Cet infléchissement est révélateur d'une époque dans laquelle Eschyle, comme d'ailleurs Sénèque, se trouvent relégués aux antipodes d'une conception classique, aristotélicienne, de la tragédie, dont les modèles sont Sophocle et Euripide. Un dramaturge britannique pouvait être réceptif à ce théâtre peu classique. Dès lors, les modèles spectaculaires sont Shakespeare et Sénèque; les passions tragiques sont l'horreur et le monstrueux, et non pas la crainte. Cette manipulation est d'autant plus aisée et naturelle que la langue d'arrivée, le latin, oblige pratiquement Stanley à garder Sénèque sous la main; surtout, il ne s'agit pas, contrairement à celles de Lefranc de Pompignan ou Du Theil, de traductions destinées au grand public, mais de travaux savants - ce qui explique le caractère scrupuleux avec lequel l'esprit d'Eschyle est préservé, mais aussi sans doute le fait qu'il soit moins difficile à Stanley d'acclimater un auteur jugé trop obscur, trop simple et trop hardi par les Français, du moins dans les ouvrages en langue vernaculaire qui

26 Stanley, Agamemnon, v. I3ı7, p. 382-383.

27 Traduction Mazon arrangée, v. I3o8, Agamemnon, p.57; Mazon traduit étonnamment

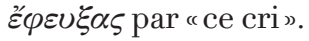

28

Sénèque, Agamemnon, v. 246. 
lui sont consacrés (par exemple, les résumés qu'en donne le père Brumoy dans sa première édition ${ }^{29}$ ).

J'aimerais, dans un deuxième temps, analyser la traduction de ces choix poétiques lorsqu'il s'agit de traiter un sujet spectaculaire mais problématique comme le meurtre entre parents. Certes, le matricide ou le meurtre des enfants est un sujet qui convient bien à une pièce écrite dans l'esprit sénéquien; mais ils font l'objet d'une certaine retenue qui prouve que, pour un traducteur moderne, ils demeurent inassimilables malgré tout.

Stanley ne recule pas devant la traduction des passages les plus «hardis », pour reprendre une expression de Lefèvre cité par Brumoy, du texte eschyléen. J'en veux pour preuve les mots du chœur à l'égard d'Agamemnon, qu'elle accuse d'être un natae mactator, "sacrificateur de sa fille», qui traduit, de manière très exacte,

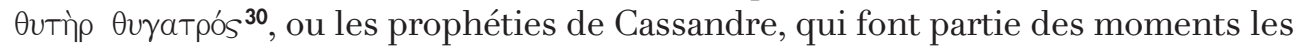
plus difficiles de la pièce. La prédiction de l'arrivée d'Oreste, en tueur de mère

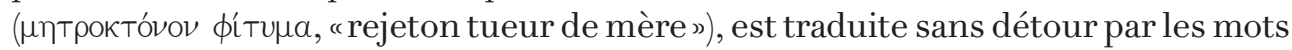
matricida progenies ${ }^{31}$. Mieux, lorsque Cassandre contemple la maison remplie de meurtres, Stanley amplifie l'horreur en ajoutant des mots à la cascade de termes utilisés par Eschyle:

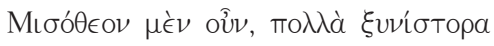

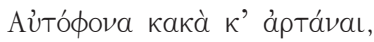

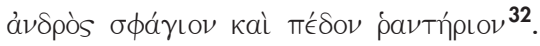

Dis plutôt une maison haïe des dieux, complice de crimes sans nombre, de meurtres qui ont fait couler le sang d'un frère, de têtes coupées [plutôt: de pendaisons]... un abattoir humain au sol trempé de sang ${ }^{33}$ !

Porro in domum diis invisam, multorum consciam

Mutuae caedis malorum, invices scelerum et suspendiorum,

Viri macellum et solum respersum sanguine.

Bien plus, dans une maison haïe des dieux, complice de maux en nombre, de meurtres réciproques, de crimes et de pendaisons alternatives, un abattoir humain et un sol inondé de sang.

29 Pierre Brumoy, Théâtre des Grecs, Paris, Rollin, Iך3o, «Discours sur le théâtre », t. I, xvj; "Agamemnon », t. II, p. I85.

30 Stanley, Agamemnon, v. 233.

31 Ibid., v. I9o, p.38o-38I.

32 Ibid., v. Io99-IIOI, p.368-369.

33 Mazon, Agamemnon, v. Iogo-Iog2, p.49. 
Le vers ııoo, qui comporte l'idée de crimes dans la même maison et de pendaisons, se trouve - une fois n'est pas coutume - glosé et développé sur trois termes par Stanley, qui insiste, par invices scelerum, sur la chaîne de crimes opérant dans le palais des Atrides - c'est le mot utilisé par la Clytemnestre de Sénèque pour parler de la chaîne de meurtres qui se succèdent dans le palais des Atrides, scelera semper sceleribus vincens domus ${ }^{34}$. La comparaison avec la traduction de Lefranc de Pompignan souligne la précision du travail de Stanley:

O palais, que les dieux détestent! Lieu souillé par tant de forfaits et de morts tragiques! Maison de carnage et de sang ${ }^{35}$ !

Lefranc de Pompignan abandonne l'image de l'abattoir, et il renonce aux détails du texte grec, au profit d'une expression très générale empruntée au domaine poétique, “morts tragiques ». C'est clairement un affaiblissement du texte, de la part d'un traducteur pourtant renommé pour son honnêteté envers le texte original. Du Theil, s'il retrouve un terme concret grâce à l'expression «réceptacle de sang ${ }^{36}$ », ne peut non plus conserver l'image trop crue de l'abattoir. Stanley, contrairement à ce que feront les traducteurs français $\mathrm{du} \mathrm{XvIII}^{\mathrm{e}}$ siècle, ne recule pas devant l'horreur, qu'il amplifie même parfois.

Cependant, on peut noter à quelques reprises de légères préventions concernant les meurtres horribles qui déchirent les Atrides. Ainsi, dans la relation que le chœur fait du sacrifice d'Iphigénie, Stanley ajoute l'intervention de Diane et

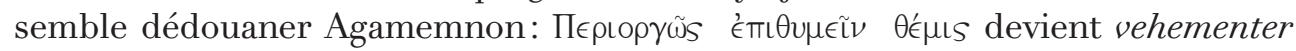
cupida est Diana ${ }^{37}$. Ce n'est plus la justice qui, en Agamemnon, introduit le souhait d'égorger sa fille, mais le désir d'une Diane qui ressemble, pour l'occasion, à une divinité vengeresse. Cette lecture est permise par le rapprochement avec les Métamorphoses, citées dans le commentaire. La déesse y est alors explicitement tenue pour responsable du meurtre d'Iphigénie: sanguine virgineo placandam virginis iram esse $\mathrm{dea}^{38}$, ce qui dédouane le père de toute faute à l'encontre de sa fille.

Quelques vers plus loin, une autre interprétation permet d'infléchir très légè-

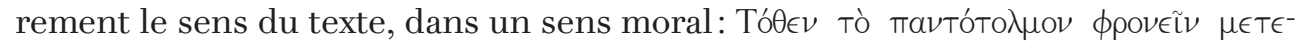
' $\gamma \nu \omega^{39}$, traduit chez Paul Mazon par «il est prêt à tout oser, sa résolution désormais est prise », devient unde supra modum sapere poenituit mortales. L'interprétation

34

Sénèque, Agamemnon, v. I69.

Lefranc de Pompignan, Tragédies d'Eschyle, Paris, Saillant et Nyon, i77o, p. 263.

Du Theil, Agamemnon, dans Pierre Brumoy, Théâtre des Grecs, Nouvelle édition, tome II, Paris, Cussac, I785, p. 8I.

Stanley, Agamemnon, v. 224-225, p.3ı-3I9.

Ovide, Métamorphoses, XII, édité par G. Lafaye, Paris, Les Belles Lettres, I93o, v. 28-29.

Stanley, Agamemnon, v. 229-23o, p.3ı8-3ig; v. 220-22I chez Mazon. 
du verbe $\mu \varepsilon \tau \alpha \gamma \iota \gamma \nu \omega ́ \sigma \varkappa \omega$ comme un verbe impersonnel me semble complètement exclue; pourtant, en choisissant cette option, Stanley peut non seulement éviter d'insister sur la dureté du choix d'Agamemnon, mais également annoncer de possibles regrets à venir pour cet acte. Le même Agamemnon avait utilisé, quelques vers plus tôt, le terrible verbe laceravero (pour $\delta \alpha^{\prime} \xi_{\omega}{ }^{40}$, déchirer), insistant sur la difficulté, pour lui, d'accomplir le geste demandé. Son émotion était d'autant plus sensible que Stanley rajoute meae devant domus ornamentum, alors que le possessif était absent du texte grec: l'amour paternel, ici, affleure alors qu'il demeure presque absent du texte grec. L’ensemble de ces légers infléchissements permet de faire d'Agamemnon une figure plus humaine, un père plus acceptable pour les lecteurs modernes.

Dans l'ensemble, le thème de la mort d'Iphigénie est traité dans un registre qui n’est pas celui de l'horrible, mais du pathétique:

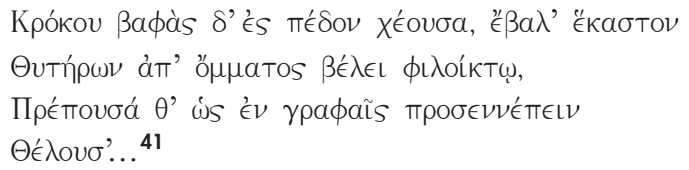

Mais, tandis que sa robe teinte de safran coule sur le sol, le trait de son regard va blesser de pitié chacun de ses bourreaux. Elle semble une image, impuissante à parler... ${ }^{42}$

At illa, croci tincturas in solum fundens, feriebat unumquemque

Sacrificorum ab oculis (emisso) jaculo misericordiam creante, Decora sicut in picturis (apparet,) alloquique volens...

Et elle, laissant tomber au sol les choses teintées de safran [sang ou robe, Stanley conserve l'ambiguïté du texte grec], elle frappait chacun des sacrificateurs d'un trait émis par ses yeux, faisant naître la pitié, elle apparaît belle comme sur un tableau, voulant parler...

Stanley attire l'attention sur ces quelques vers déjà très picturaux chez Eschyle, en les ouvrant par le contrastif at illa, qui alourdit l'effet dramatique déjà construit par Eschyle et l'opposition entre la jeune fille et le groupe de ses sacrificateurs. Les

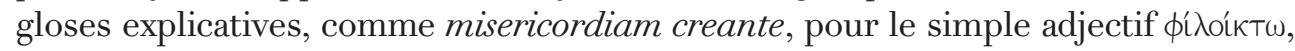
soulignent à quel point le spectateur est présent à l'esprit de Stanley, qui amplifie le dispositif de spécularité déjà proposé par Eschyle. L'intervention du verbe apparet, de même, construit la scène sous les yeux du lecteur. Le commentaire souligne,

\footnotetext{
$40 \quad$ Ibid., v. $2 \mathrm{I} 6$.

41 Ibid., v. 247-249, p.320-32I.

42 Mazon, Agamemnon, v. 238-243, p. I8.
} 
au sujet du vers vestibus involutam ${ }^{43}$, l'importance du regard: il s'agit de demeurer honestius, respectable, afin de pouvoir susciter la pitié - et non, comme le caractère pictural du passage pourrait le laisser entrevoir, un regard érotisé sur une jeune fille nue, proie de ses bourreaux. Ce souci fait écho au récit de la mort de Polyxène par Ovide, qui tient à expliciter combien la jeune fille a soin de casti decus servare pudo$r i s^{\mathbf{4 4}}$ - et la source ovidienne est, en l'occurrence, le gage d'une tonalité éminemment pathétique. La comparaison de ces vers de Stanley avec ceux traduits par Lefranc de Pompignan et Du Theil est frappante. Lefranc ne conserve pas l'image du tableau ${ }^{45}$; quant à Du Theil, il affaiblit le texte en rendant l'image plus abstraite, par l'expression un peu figée: “Belle comme les merveilles de l'art ${ }^{46}$.» Aucun de ces deux traducteurs ne traduit d'ailleurs correctement le participe $\theta \dot{\epsilon} \lambda$ ov $\sigma \alpha$, pour lequel on trouve dans les deux textes français « elle semble»: l'image d'une beauté muette parce qu'incapable de parler, luttant contre le destin qui la contraint au silence, est abandonnée, au profit d’une simple illusion de réel donnée par le tableau. De ce point de vue, la traduction de Stanley est à la fois bien plus pathétique et bien plus fidèle à l'original.

Je voudrais à présent examiner plus précisément le vocabulaire de la famille, tel que l'utilise Stanley: ses choix de traduction peuvent souligner combien sa vision de la famille est orientée par des préoccupations modernes. C'est ainsi que, pour

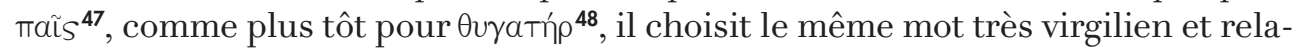
tivement peu précis de nata, dans lequel on peut voir la nature, au sens de naissance - et non la notion de petit enfant que comportait naĩs, pour lequel on aurait

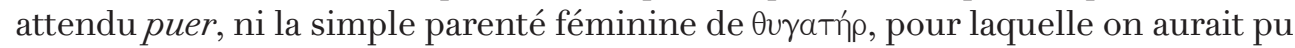
avoir filia. Nata insiste sur le lien de naissance qui unit Agamemnon à sa fille, un

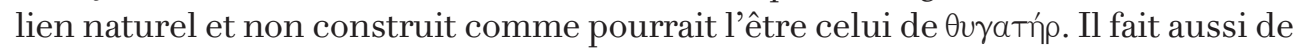
la jeune fille un "petit», comme on pourrait le dire d'un petit d'animal. Avec nata, on entre dans un domaine naturel, qui amplifie la faute d'Agamemnon pour des Modernes: il a osé porter la main contre son propre sang, contre ce qui était sorti de lui - et non pas, seulement, contre la fille qui faisait partie de sa famille pour le reste de la société. Pour traduire les douleurs de l'enfantement, $\omega \dot{\delta} s^{49}$, Stanley choisit partus, qui oublie la dimension douloureuse de l'acte, au profit d'un mot qui sort l'attachement de la mère pour sa fille du contexte tragique dans lequel elle

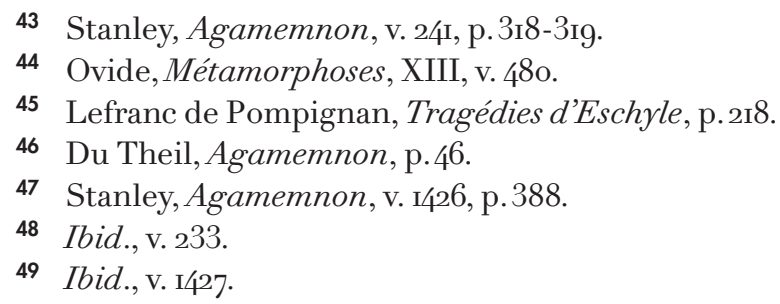


l'envisage, comme une rétribution pour ses peines, et en fait au contraire un fruit, un petit être à couver comme une plante fragile. Fruit, Iphigénie le devient aussi par l'utilisation du terme germen, pour traduire - de manière très heureuse - le

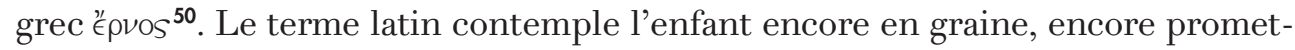
teuse; le terme grec, lui, voit en elle un rejet de l'arbre que sont ses parents, une jeune pousse destinée à grandir, mais déjà développée. Tuée alors qu'elle n’est qu'à l'état de germen, Iphigénie n'a pas connu le développement que le mot épvos lui accordait malgré tout, faisant d'elle la jeune fille séduisante qu'elle a été. Enfin, dans le mot latin, est contenue une promesse de fruits, l'espoir d'une mère dans sa progéniture: l'espoir que la fille devienne mère à son tour, et assure une descendance à ses parents. Le vocabulaire dédié aux enfants contribue à créer, autour d'Iphigénie, un contexte d'attachement naturel, presque animal, qui n'existe pas, me semble-t-il, dans le texte grec.

Or, les enfants, le texte grec le dit, sont garants de la foi entre les parents :

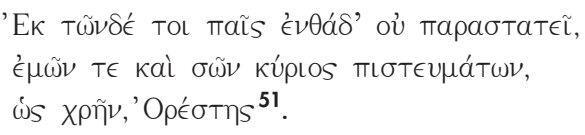

Et c'est aussi pourquoi ton fils n'est pas ici, comme il eût convenu, Oreste, le garant de $[\text { ma fidélité et de la tienne }]^{52}$.

\section{Ob haec quidem filius hic non astat, \\ Meae et tuae fidei pignus, \\ Sicut oportuit, Orestes.}

C'est à cause de cela, oui, que ton fils n'est pas là, le gage de ma foi et de la tienne, comme il aurait fallu, Oreste.

De manière révélatrice, ici, Stanley choisit le terme de filius pour traduire пaĩs : lorsqu'il s'agit du fils, c'est la filiation qui est retenue, et non le caractère pathétique du petit enfant. Le contexte invite d'ailleurs à ce choix, puisqu'il s'agit ici d'expliquer combien les enfants sont, presque juridiquement, un garant du mariage de leurs parents (cette idée se trouve également dans les discours juridiques grecs ${ }^{53}$ ). Surtout, le terme de kúpıos, très marqué sur le plan juridique, cède la place à pignus, un terme également juridique, mais dont les implications sont

50 Ibid., v. I534, p.394-395.

51 Ibid., v. 886-888, p.356-357.

52 Traduction Mazon modifiée, v. 877-879, p.4I.

53 Voir par exemple Lysias, Sur le meurtre d'Ératosthène, 6-7, dans Discours, I, éd. L. Gernet et M.Bızos, Paris, Les Belles Lettres, I943 [I924]. Voir également Aristote, Éthique à 
différentes de celles du mot grec. Chez Eschyle, les enfants, en tant que кúpıo, sont constitués comme des personnes morales exerçant une autorité souveraine sur leurs parents; chez Stanley, le mot de pignus les réduit à l'état de simples objets déposés en caution pour assurer le succès d'une entreprise - ce qui va dans le sens d'une réification des enfants, dans la traduction latine. Le choix du mot fides, lui, pour traduire le très concret $\pi \iota \sigma \tau \epsilon u ́ \mu \alpha \tau \alpha$, pourrait permettre de rétablir l'équilibre entre abstrait et concret; cependant, il place l'abstraction et donc l'autorité morale dans les parents, dont il s'agit de préserver les vœux, et non plus dans les enfants qui pouvaient constituer un garant de ces actions : le regard sur l'enfant a changé, avec Stanley, et l'on conçoit désormais le fils ou la fille comme le petit être à protéger, non comme l'enjeu juridique d'un mariage qu'il pourra venir sauver s'il le faut ${ }^{54}$.

En lien avec ces modifications dans le vocabulaire utilisé pour parler de l'enfance, est introduite une réflexion sur la femme, qui prend une importance cruciale dans le cadre de la trilogie d'Eschyle. J'en prendrai pour exemple la dernière scène, la confrontation entre Égisthe et le chœur. Là où le texte varie peu ses appellations (la femme, dans ce passage, c'est toujours ruvì), Stanley ressent le besoin de faire intervenir deux termes : mulier ${ }^{55}$ et ses composés muliebre ${ }^{56}$, muliebris ${ }^{57}$, ou foemina ${ }^{58}$ - sans raison apparente, puisqu'il s'agit la première fois de qualifier Égisthe, une femme qui reste à la maison; puis pour muliebre, de la ruse revenant à la femme; enfin, pour muliebris, de l'avis d'une femme; quant à foemina, que

Nicomaque, VIII, ı6ı b I8-I9 et II62 a 27-29, trad. R. BodÉüs, Paris, GF Flammarion, 2004, p. 442 et 445 .

54 Sur les bouleversements qui affectent le regard sur l'enfance aux XvII ${ }^{\mathrm{e}}$ et $\mathrm{XvIII}^{\mathrm{e}}$ siècles, voir Ph. AriÈs, L'Enfant et la vie familiale sous l'Ancien régime, Paris, Seuil, I975 [196o], notamment p.299-3o7; ce travail a été continué par J.GéLIs, "L'individualisation de l'enfant », dans Histoire de la vie privée, III, De la Renaissance aux Lumières, sous la direction de Ph. Ariès et G.Duby, Paris, Seuil, ig85, p.3o3-3i8; A. Burguière, “Les cent et une familles de l'Europe ", dans Histoire de la famille, III, Le choc des modernités, sous la direction de A. Burguière, C. Klapisch-Zuber, M. Segalen et F. Zonabend, préface de J. Goody, Paris, Armand-Colin, I986, p. 2I-I22; É. Badinter, L'amour en plus. Histoire de l'amour maternel (xVII ${ }^{\mathrm{e}}-\mathrm{XX} \mathrm{x}^{\mathrm{e}}$ siècle), Paris, Flammarion, I980, notamment deuxième partie, chapitre I, p. I/II-I94; E. SсHовтER, Naissance de la famille moderne, XVIII ${ }^{\mathrm{e}}$ - XX $\mathrm{X}^{\mathrm{e}}$ siècle, traduit par Serge Quadruppani, Paris, Seuil, I977, p. 208-253; Cl.Bernard, Ch. Massol et J.-M.Roulin, introduction à l'ouvrage collectif Adelphiques, 20IO, p. Io; Cl. Bernard, Penser la famille au dix-neuvième siècle (1789-I870), Saint-Étienne, PusE, 2007, p.32-39. Stanley, Agamemnon, v. I634, p.400-40I.

56 Ibid., v. I645, p. 402-403.

57 Ibid., v. 1670.

58 Ibid., v. 1653. 
l'on aurait plutôt imaginé traduisant $\theta \eta \lambda \epsilon i ́ a$, en référence au genre, il est utilisé lorsque le chœur s'étonne qu'Égisthe ait laissé une femme frapper le héros. En apparence, peu de différence: le mot est toujours utilisé en opposition directe ou sous-jacente à l'homme, qui aurait dû prendre l'action ou n'aurait pas usé de ruse. On peut peut-être établir une différence d'utilisation entre des mots qui font référence aux comportements attendus d'une femme (mulier), et ceux qui évoquent l'action menée par une faible femme (foemina). Notons que le grec utilise, dans la conclusion du coryphée, $\theta \eta \lambda \epsilon i ́$, dans son sens animal de femelle, ici inséparable de

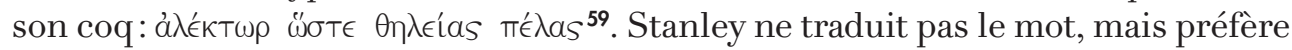
le remplacer directement par le féminin du Gallus, faisant de Clytemnestre une poule - ce qu'elle n'était pas complètement chez Eschyle: tanquam Gallus prope gallinam. La femme subit alors un traitement encore plus dégradant que dans l'original grec.

Lorsqu'il est question des sentiments de la femme pour son amant, Stanley opère un détour étrange et peu naturel, peut-être légèrement pudique, à l'égard de ce que confesse Clytemnestre: la reine grecque avoue ce qu'elle nomme ses

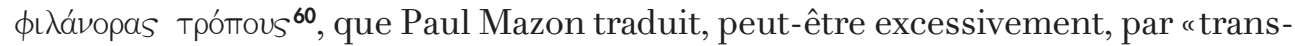
ports amoureux». Il est évident, malgré tout, que тро́тоs fait référence à des attitudes, à un comportement plus qu'à un sentiment. Stanley traduit, lui, en termes de sentiments: Viri amans ingenium meum. L'utilisation du mot vir, par ailleurs, contribue à donner une forme de légitimité à Égisthe, promu quasiment au rang d'époux - ou du moins aux sentiments de Clytemnestre pour celui qu'elle considère, dans cette expression, comme tel. La femme agit alors selon ce que lui demande son devoir, en épouse aimante, et son esprit se soumet moralement à cette règle dictée par la société61.

Le travail de Stanley se signale par la probité et le respect avec lesquels il traite le texte source: le traducteur livre une version scrupuleuse, dans laquelle les travaux de ses prédécesseurs permettent de donner du texte un mot à mot respectant l'ordre, le rythme et les nuances de l'original. Ce travail fait date pour l'histoire de la réception d'Eschyle. Pourtant, les quelques libertés que sa pratique scrupuleuse laisse à Stanley permettent de souligner, à la marge, des infléchissements dans deux directions. La première est le modèle spectaculaire de Sénèque, à l'aune duquel on lit Eschyle, fort peu connu au demeurant avant le travail de Stanley, et que l'on juge étranger à toute tentative d'apprivoisement par les règles de dramaturgie classique - il est donc de ce fait immédiatement rejeté du côté de

59 Ibid., v. 168o, p.404-405.

60 V. 865, p.356-357; v. 856 chez Mazon.

61 Réminiscence, peut-être, de Sénèque, Agamemnon, v. 239: “Amor conjugalis vincit.» 
Sénèque, auquel il est d'ailleurs accolé dans l'édition de Brumoy, et de Shakespeare. Le choix du latin comme langue d'arrivée explique aussi, sans doute, cette familiarité de Stanley avec des modèles comme celui de Sénèque, soutenu par des références à Ovide ou Virgile également. Cette première tendance entre en contradiction avec une deuxième influence qui, malgré la liberté dont il fait preuve, pèse sur Stanley: certes, le contexte de traduction savante et en latin lui permet, contrairement à Lefranc de Pompignan, de demeurer très près du texte source; malgré tout, quelques réticences se font sentir, et surtout quelques infléchissements dans le choix du vocabulaire montrent combien la conception de la famille, à l'époque moderne, se démarque de ce qu'elle peut être pour Eschyle et combien les modèles grecs sont de ce fait difficiles à transcrire dans l'Europe moderne.

\section{Marie Saint Martin}

Professeur en CPGE, membre rattachée au CRLC

Saintmartin.marie@orange.fr 